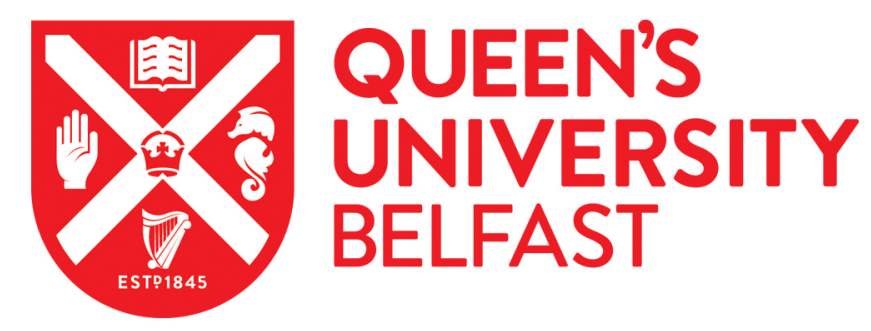

\title{
A rigorous and accurate contrast spectroscopy for ultimate thickness determination of micrometre-sized graphene on gold and molecular sensing
}

Katzen, J., Velický, M., Huang, Y., Drakeley, S., Hendren, W., Bowman, R. M., Cai, Q., Chen, Y., Li, L. H., \& Huang, F. (2018). A rigorous and accurate contrast spectroscopy for ultimate thickness determination of micrometre-sized graphene on gold and molecular sensing. ACS Applied Materials and Interfaces, 1-9. https://doi.org/10.1021/acsami.8b01208

Published in:

ACS Applied Materials and Interfaces

Document Version:

Peer reviewed version

Queen's University Belfast - Research Portal:

Link to publication record in Queen's University Belfast Research Portal

Publisher rights

Copright 2018 American Chemical Society. This work is made available online in accordance with the publisher's policies. Please refer to any applicable terms of use of the publisher.

\section{General rights}

Copyright for the publications made accessible via the Queen's University Belfast Research Portal is retained by the author(s) and / or other copyright owners and it is a condition of accessing these publications that users recognise and abide by the legal requirements associated with these rights.

\section{Take down policy}

The Research Portal is Queen's institutional repository that provides access to Queen's research output. Every effort has been made to ensure that content in the Research Portal does not infringe any person's rights, or applicable UK laws. If you discover content in the Research Portal that you believe breaches copyright or violates any law, please contact openaccess@qub.ac.uk. 


\section{A rigorous and accurate contrast spectroscopy for}

\section{ultimate thickness determination of micrometre-sized}

\section{graphene on gold and molecular sensing}

Joel M. Katzen', Matěj Velický, Yuefeng Huang', Stacey Drakeley’, William Hendren , Robert M. Bowman, Qiran Cai , Ying Chen , Lu Hua Li ${ }^{\ddagger}$, and Fumin Huang**

$\dagger$ School of Mathematics and Physics, Queen’s University Belfast, BT7 1NN, United Kingdom

\$ Institute for Frontier Materials, Deakin University, Waurn Ponds, Victoria 3216, Australia

KEYWORDS: graphene; optical contrast; gold; airborne contamination; molecular sensor

\section{ABSTRACT}

The thickness of graphene films can be accurately determined by optical contrast spectroscopy. However, this becomes challenging and complicated when the flake size reduces to the micrometre scale, where the contrast spectrum is sensitively dependent on the polarization and incident angle of light. Here we report accurate measurement of the optical contrast spectra of micrometre-sized few-layer graphene flakes on Au substrate. Using a high-resolution optical microscopy with a 100x magnification objective, we accurately determined the layer numbers of flakes as small as one micrometre in lateral size. We developed a theoretical model to accurately take into account the appropriate contribution of light incident at various angles and polarizations, which matched the experimental results extremely well. Furthermore, we demonstrate that the optical contrast spectroscopy is highly sensitive to detect the adsorption of a submonolayer airborne hydrocarbon molecules, which can reveal whether graphene is contaminated. Though 
the technique was demonstrated on graphene, it can be readily generalized to many other two-dimensional (2D) materials, which opens new avenues for developing miniaturized and ultrasensitive label-free molecular sensors.

\section{INTRODUCTION}

Gold is widely used as the substrate material in many graphene devices, due to its superior optoelectronic properties and chemical stability. However, there has been little experimental investigation on the optical contrast of graphene films on Au substrates. Here we report accurate measurement of the optical contrast spectra of few-layer graphene flakes on bulk Au. We used a high-resolution optical microscopy with a $100 \times$ magnification objective, accurately determining the thickness of flakes as small as one micrometre in lateral size, which are highly desired in many applications. The results are in excellent agreement with theoretical calculations and confirmed by Raman and AFM measurements. Furthermore, we demonstrate that the optical contrast spectroscopy is sensitive enough to detect the adsorption of a sub-monolayer airborne hydrocarbon molecules, which can reveal whether graphene is contaminated and opens the opportunity to develop miniaturized and ultrasensitive molecular sensors.

Graphene has attracted a lot of interest since its discovery in $2004^{\prime}$, owing to a plethora of extraordinary performance in electronic, mechanical, thermal and optical properties. It is a zero-gap semiconductor due to the fact that the conduction and valence bands meet at the Dirac point ${ }^{2}$, and has electron mobilities of more than $15000 \mathrm{~cm}^{2} . \mathrm{V}^{-1} \cdot \mathrm{S}^{-1}$ at room temperature ${ }^{3}$. As a result, it has the lowest resistivity $\left(\sim 10^{-6} \Omega . \mathrm{cm}\right)$ of any known conductive material at room temperature ${ }^{4}$. It also has exceptional mechanical strength and thermal conductivitys. These remarkable properties make graphene one of the most exciting materials widely investigated across multiple areas, including energy, electronics, photonics, and sensors ${ }^{6,7}$. For graphene devices, the choice of underlying substrate is critical and impacts the functionality of the device in many aspects, e.g., modulating the electrical and thermal conductivities, doping and affecting the Raman and fluorescence spectroscopy, and modifying the electrochemical properties ${ }^{8}$. Many applications 
require graphene to be deposited on metallic substrates, such as copper ${ }^{9}$ nickel ${ }^{10}$, silver $^{11}$ and gold ${ }^{12-14}$, to achieve optimal electronic, plasmonic, catalytic and sensing functionalities ${ }^{15-17}$. Gold in particular is a popular choice of metallic material, due to its excellent optoelectronic properties and chemical stability. On the other hand, much of the research and applications require high quality pristine graphene films, which are often produced by mechanical exfoliation, resulting in small flakes of a few microns or even less. Such small films hold realistic hopes for graphene commercialization in the near future. Despite important advancement has been made in the CVD synthesis of graphene films, it is still extremely challenging to fabricate uniform and defect-free high-quality large-area graphene films, which poses as a significant barrier for the commercialization of graphene devices. By contrast, micrometre-sized high quality graphene films are readily to make and can be fabricated in large scales, which have been exploited to develop a wide range of miniaturized nanodevices, such as high frequency transistors, ultra-broadband photon detectors, low-threshold mode-locked lasers, ultrasensitive molecular sensors, and even in spintronics $^{1820}$. Hence, accurate determination of the thickness of micrometre-sized graphene films on gold substrates is essential in graphene research and technology development.

Several methods are commonly used to identify the number of atomic layers in a graphene flake, including atomic force microscopy (AFM), Raman spectroscopy and optical contrast spectroscopy ${ }^{21}$. AFM is a low-throughout technique, taking up to several hours to produce a detailed enough image from which the important data can be extracted. It is also subject to the influence of a number of factors, such as humidity, surface roughness and contamination. Raman spectroscopy is much faster and can probe the flake's thickness non-destructively, based on the ratio of the G- and 2D-peaks, as well as the position and shape of the 2D-peak ${ }^{2}$. However, these spectral features can be influenced by many factors, including temperature ${ }^{23}$, strain $^{24}$, and doping ${ }^{25}$, therefore sometimes giving ambiguous results for the number of layers present. Compared to AFM and Raman spectroscopy, optical contrast spectroscopy has a number of distinct advantages: it is easy to implement, fast, noninvasive, and less affected by the aforementioned factors. As such, it has become an attractive technique for identifying the layer thickness of graphene and a wide variety of other 2D materials ${ }^{26228}$. 


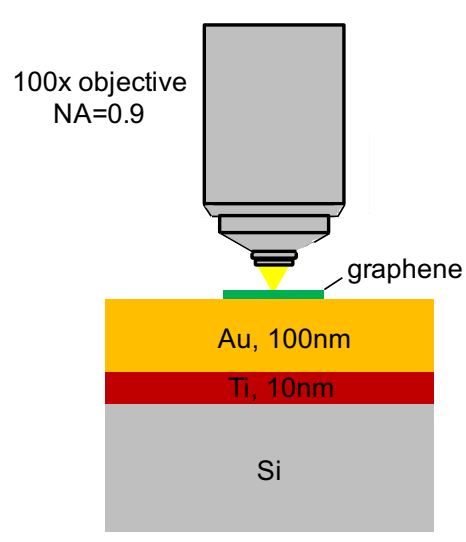

(a)

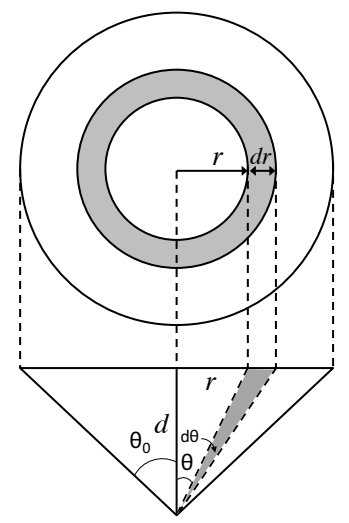

(b)

Figure 1. Schematic showing the experimental configuration (a) and the diagram of the incidence and focusing of light (b). The circle in the top panel of $\mathbf{b}$ refers to the incident aperture of the objective (top view). The triangle in the bottom panel indicates the focusing cone of light through the objective. The correspondence between the incident annular ring and the focusing cone is indicated by shaded areas.

Optical contrast of graphene films on oxidized silicon substrates has been studied extensively ${ }^{29}$. In contrast, very limited investigation has been carried out on metallic surfaces. Previously there was a theoretical investigation of the contrast of graphene on thin Au films, based on simple Fresnel reflectivity formulae at normal incidence ${ }^{30}$. However, there have been no experimental investigations of the optical contrast of graphene films on gold surfaces. Here we provide detailed experimental measurements, for the first time, of the optical contrast of few-layer (1-5 layer) graphene flakes on a bulk Au surface, using high-resolution optical microscopy with a $100 \times$ magnification objective (Figure 1), which enables us to accurately identify the thickness of flakes as small as half micrometre (the diffraction limit of the $100 \times$ magnification objective) in lateral size.

The experimental results obtained are in excellent agreement with theoretical calculations from a modified model, taking into account the effects of different incident angles and polarizations, which become significant with the use of a high numerical aperture objective. The accurate determination of the layer numbers was validated by independent AFM and Raman measurements. In addition, we found that the 
high accuracy and sensitivity of the optical contrast spectroscopy enabled the detection of the adsorption of a sub-monolayer contamination molecule. The optical contrast of freshly-prepared samples matched perfectly with that predicted by the theoretical model, whereas samples exposed to ambient air exhibited an increase of the contrast with time, as a result of the adsorption of airborne hydrocarbon molecules. This can reveal whether graphene is contaminated and could be exploited to develop miniaturized and highly sensitive optical sensors.

\section{RESULTS AND DISCUSSION}

Figure 1 shows the experimental configuration. Au film of $100 \mathrm{~nm}$ was deposited on $\mathrm{Si} / \mathrm{SiO}_{\mathrm{x}}$ wafer through magnetron sputtering, with a $10 \mathrm{~nm}$ Ti adhesion layer (Figure 1). The Au film is thick enough to be equivalent to a bulk material. Less than $1.3 \%$ light can transmit through the Au film, so light reflected from the underlying substrate is negligible. To minimize the chance of contamination, graphene flakes were exfoliated onto Au films immediately after they were taken out of the sputtering chamber using the scotch tape method, and optical reflectance measurements were conducted soon afterward.

Figure 2 shows the bright-field optical images of the exfoliated graphene films (1-5 layers, details about how the layer numbers are determined will be introduced later). Without using any optical filter, flakes of three layers and above are clearly distinguishable (Figure 2a-b), while the monolayer and bilayer flakes are only faintly visible. The contrast of the flakes is considerably enhanced when a $490 \mathrm{~nm}(10 \mathrm{~nm}$ bandpass width) band-pass filter is placed in front of the light source (Figure 2c-d). The bilayer flakes now are clearly visible. The visibility of the monolayer flake, though still faint, is also improved, which now can be visualized when the image is converted to greyscale and the contrast is enhanced by a factor of two (Figure 2e). 
To accurately quantify the optical contrast of the graphene flakes, we measured the reflectance spectra on bare Au surfaces and on graphene flakes, respectively, and calculated the contrast spectra using equation 3 (see MATERIALS AND METHOD). The results are shown in Figure 3a (red curves).

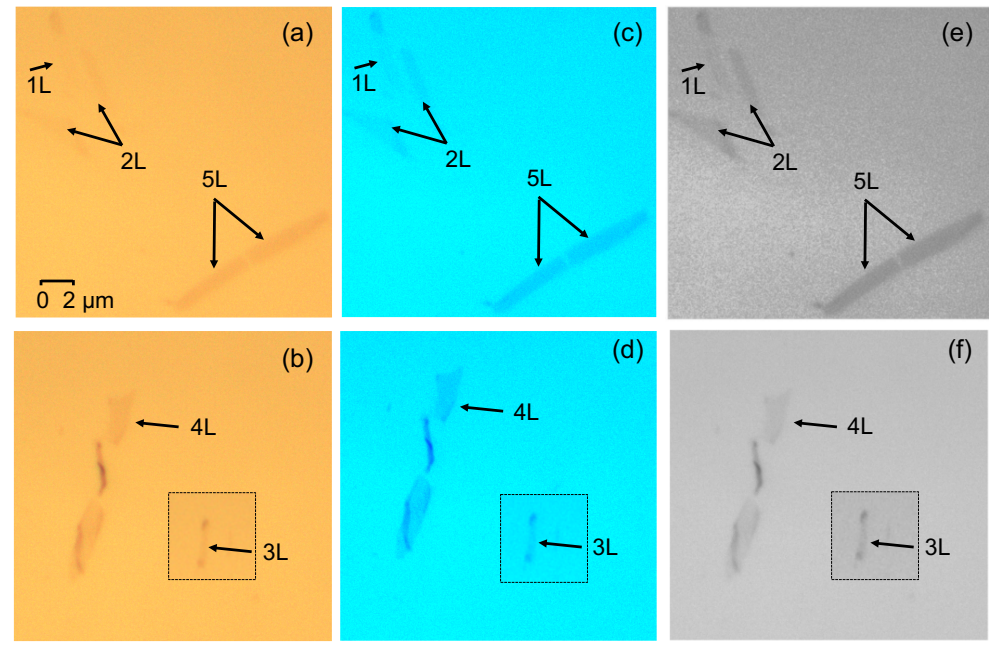

Figure 2. (a-b) Bright-field optical images of 1-5 layers graphene flakes without any optical filter, (cd) with a $490 \mathrm{~nm}$ optical filter (10 nm bandwidth), and (e-f) greyscale images of (c-d). The contrast in $\mathbf{e}$ is enhanced by a factor of two for a better view of the monolayer film. The $3 \mathrm{~L}$ on the sample and superimposed in the image. The inset of the $3 \mathrm{~L}$ film were taken at a different location on the sample and superimposed here.

It is evident that the contrast increases with the number of graphene layers. The maximum contrast occurs around $500 \mathrm{~nm}$, which shifts very little between the 1 to 5 layer films. This explains why images filtered by a $490 \mathrm{~nm}$ optical filter show significantly enhanced optical contrast (Figure 2c-d).

In previous research, simple Fresnel reflection formula at normal incidence is often used to calculate the reflection and the contrast spectra ${ }^{3 x_{3032}}$. This produced reasonably good match with experimental results, through tailoring the refractive index (RI) of graphene, but resulting in various different RI values. Generally, reflectance is dependent both on the incident angle and the polarization of light. The difference may not be notable when the incident angle is small, such as in the case of a low magnification objective. However, the impact of the incident angle and polarization will become pronounced with increasing incident angle. The reflectance at large incidence angles could be significantly different from that of 
normal incidence. To illustrate this, we calculated the reflectance of the bare Au surface (no graphene) and the optical contrast of monolayer graphene on $100 \mathrm{~nm} \mathrm{Au}$ (Figure 1a) at various incident angles and polarizations (Figure 4), based on the standard transfer matrix method ${ }^{31}$. The reflectance is almost constant up to $20^{\circ}$, but changes markedly with increasing incident angles (Figure $4 \mathrm{a}-\mathrm{b}$ ). As a result the contrast is inhomogeneous across incident angles and polarizations (Figure 4c-d). In addition, the percent-
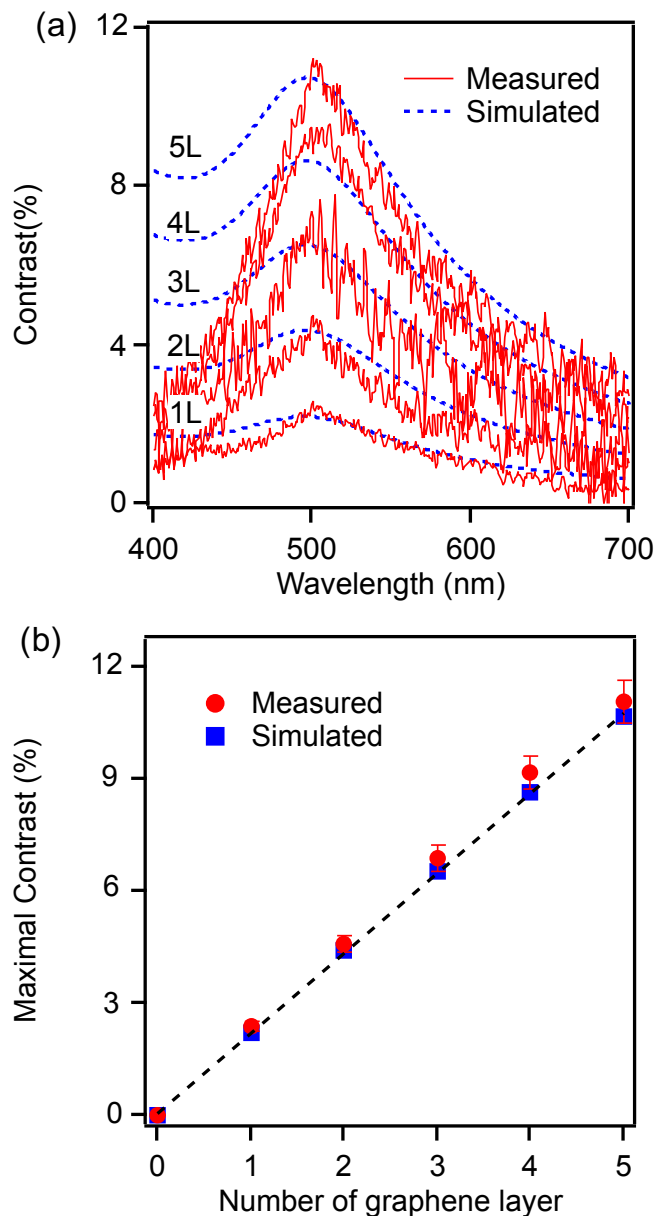

Figure 3. (a) Measured (red) and simulated (blue) optical contrast spectra of the 1-5 layers of graphene flakes shown in Figure 2. (b) The maximum optical contrasts of measured (red) and simulated (blue) results as a function of the number of graphene layers, fitted by a straight line passing through the origin point of zero contrast and zero thickness.

age of light incident at various angles is quite different. More light is incident at larger angles (Figure 1b). A high numerical aperture (NA) objective has a wide incident cone with a spanning angle of $\theta_{0}$ 
$\left(\sin \theta_{0}=\mathrm{NA}\right.$, e.g., $\mathrm{NA}=0.9, \theta_{0}=649$, as indicated in Figure $1 \mathrm{~b}$. It is thus necessary to appropriately average the contribution of various incident angles and polarizations to get the accurate reflectance and the contrast spectrum, which are given below (see Supporting Information):

$$
\begin{gathered}
R_{\text {ave }}=\frac{1}{2}\left(\bar{R}_{T E}+\bar{R}_{T M}\right) \\
\bar{R}_{T E, T M}=\frac{2 \int_{0}^{\theta_{0}} R^{T E, T M}(\theta) \tan \theta(\sec \theta)^{2} d \theta}{\left(\tan \theta_{0}\right)^{2}}
\end{gathered}
$$

$\bar{R}_{T E, T M}$ is the averaged (over all incident angles) reflectance and $R^{T E, T M}(\theta)$ is the reflectance at the incident angle of $\theta$, for TE and TM polarizations, respectively. The contrast is then calculated based on equation 3. The resulting calculated contrast spectra are shown in blue alongside the experimental data in Figure 3a. The thickness of graphene is given by $\mathrm{d}=0.335 \mathrm{~N} \mathrm{~nm}$, where $\mathrm{N}$ is the number of layers. The refractive index of graphene was taken as 2.6-1.3i, the same as that of graphite, which has been shown to produce consistent simulation results with experiments ${ }^{3032}$. The refractive index of $\mathrm{Au}$ is adopted from literature $^{33}$. There is excellent agreement between the two sets of results in the long wavelength $(\lambda>500$ $\mathrm{nm}$ ) region, especially around the peaks. We plot the measured maximum optical contrasts of the flakes together with those simulated as a function of the layer number, shown in Figure $3 \mathrm{~b}$. As can be seen, the two sets of data match extremely well. The maximum contrast is linearly proportional to the number of layers, fitted very well by a straight line (dashed line). When the linear fitting curve is extrapolated, it passes through the origin point of zero contrast and zero thickness, as would be expected in an ideal situation. This confirms that the theoretical model is effective and the samples investigated were clean and of high quality. The discrepancy between the experimental data and the simulated results at short wavelength is notable. The calculated reflectivity spectrum perfectly matches the experimental one in the long wavelength range, but deviates considerably in the short wavelength range (Figure S2, Supporting Information). This is possibly due to a multitude of factors. Firstly, the assumption that graphene has a 
constant refractive index in the visible range is not true in reality. It varies with wavelength, and this dependence becomes more pronounced in the short wavelength region ${ }^{3435}$. Secondly, the assumption there is equal amount of TE and TM polarization light may not be true either. Various optical elements (e.g., beamsplitter, fiber etc.) may not function exactly with equal efficiency for TE and TM polarizations. The reflectivity of TE and TM polarizations deviates strongly in the short wavelength region. A slight imbalance between TE- and TM-polarized light will have a considerable impact on the overall reflectivity in the short wavelength range, but negligible in the long wavelength range (Figure S2). Some other factors may also contribute to the discrepancy (see more details in SI).
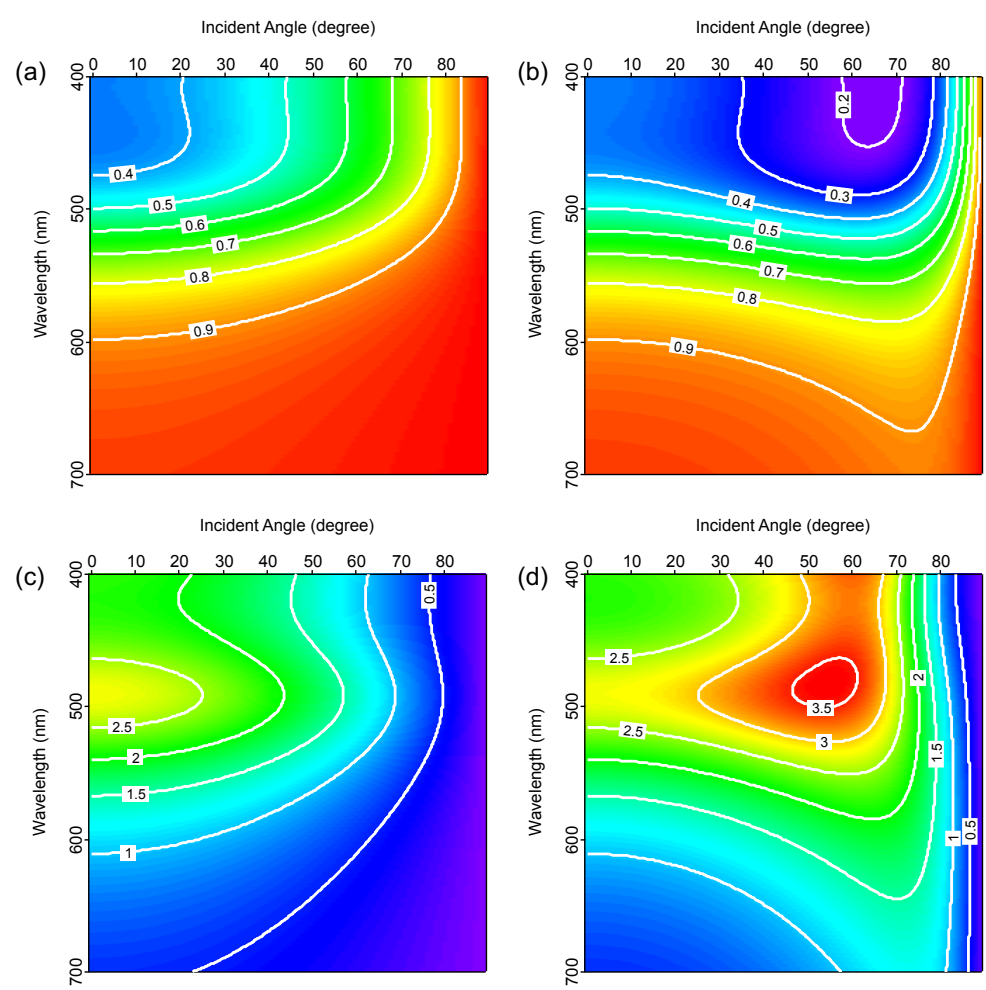

Figure 4. Calculated optical contrast and reflectivity of monolayer graphene on $100 \mathrm{~nm} \mathrm{Au}$, as functions of the incident angle and wavelength of light. (a-b) reflectance (absolute magnitude), (c-d) contrast (in percentage). The left and right panels are for TE- and TM- polarizations, respectively. Refractive index of graphene $n_{G}=2.6-1.3 i$, thickness $0.335 \mathrm{~nm}$. 
To further confirm the accurate identification of the number of graphene layers, Raman and AFM measurements were carried out. The measured Raman spectra are shown in Figure 5a. As the thickness of graphene film increases, the G-peak becomes more intense in relation to the 2D-peak. Additionally, the 2D-peak gets broader, because on thicker flakes, the graphene film experiences a double resonance relating to intermediate phonon scattering electronic state ${ }^{36}$. The intensity of the G-peak scales linearly with the film thickness (Figure 5b). These properties are characteristic features of the Raman spectrum of
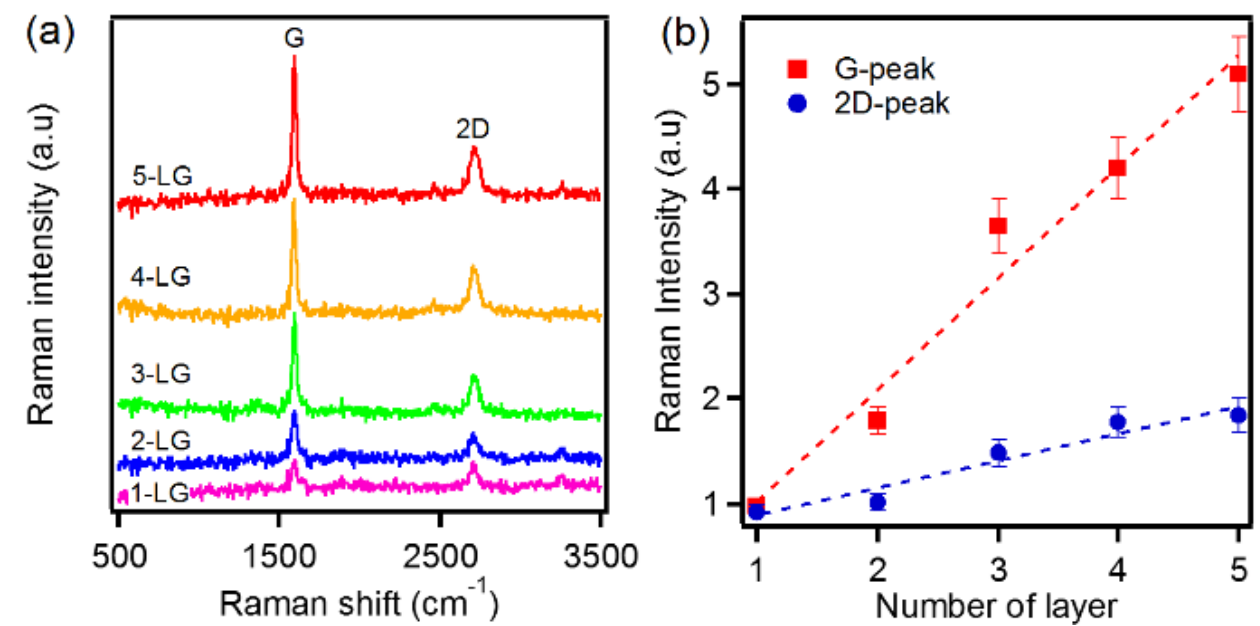

few-layer graphene ${ }^{22}$. Here the $\mathrm{G}$ mode and the 2D mode of the monolayer graphene is at a similar intensity

(Figure 5a). In normal circumstances, the 2D peak is stronger than that of the $\mathrm{G}$ peak ${ }^{22}$. However, it is known that the intensity of the $2 \mathrm{D}$ peak can be strongly affected by doping ${ }^{37}$. By contacting metallic substrates, the 2D peak can even be weaker than the $\mathrm{G}$ peak in monolayer graphene ${ }^{2538}$. This shows the limitation of the Raman spectroscopy technique as a precise tool to accurately determine the graphene thickness.

Figure 5. (a) Raman spectra measured for 1-5 layers of graphene, excited by a $532 \mathrm{~nm}$ laser. (b) Normalized Raman intensities of the G- (red) and 2D-peak as a function of the number of layers. The intensities of the G-peak and the 2D-peak of each flake are normalized to the intensities of the G- and 2Dpeaks of the monolayer film. 
Figure 6 shows the results from tapping-mode AFM measurements of the graphene flakes. Step profiles give heights of $0.56 \pm 0.10,0.75 \pm 0.15,1.1 \pm 0.15$, and $1.6 \pm 0.2 \mathrm{~nm}$ for one, two, three, and five layers respectively. The measured thickness of two, three, and five-layer graphene flakes matched quite well with the expected values within experimental uncertainties. The height of the monolayer flake is slightly thicker than expected, which is not unusual in AFM measurements. AFM results are subject to the influence of a number of factors ${ }^{390}$, such as the humidity of environment and the roughness of substrates. It has been observed experimentally that when graphene is placed on top of a rough surface, the flake acts like a tissue-sheet and follows the substrate's morphology $y^{3940}$. When the substrate's roughness is higher than the flake's thickness, the measured height is deceptively higher than the flake's true height. It is thus not surprising that the measured height of the monolayer graphene film appears slightly thicker than expected. These results unambiguously confirmed that the number of the layers determined by the optical contrast method are accurate.

Figure 3 reveals that graphene only has a modest contrast on bulk $\mathrm{Au}(\sim 2.2 \%$ maximum contrast for monolayer), much lower than that on $90 \mathrm{~nm} \mathrm{SiO} / \mathrm{Si}$ substrates where the maximum contrast of monolayer graphene reaches up to $12 \%{ }^{29}$ The low visibility of graphene on Au substrate is a result of the high reflectivity of $\mathrm{Au}$ surface (Figure S2), which is a downside shared by all reflective metals, such as $\mathrm{Ag}, \mathrm{Cu}$ and $\mathrm{Al}$.

Despite the modest optical contrast, the distinct contrast spectra of different layers and the linear dependency on the layer number make it a powerful tool to accurately identify the layer thickness of micrometre-sized graphene flakes, which are often generated by mechanical micro-cleavage method, to produce high quality pristine graphene. Compared to Raman and AFM measurements, the optical contrast method is fast, more definitive and less affected by factors such as strain, doping and temperature. In addition, the optical contrast of graphene is highly sensitive to the layer thickness, which changes drastically upon the addition of just one atomic layer of carbon atoms. Such a high sensitivity can have potential applications in many areas, for instance, to reveal whether graphene is contaminated, as when 
molecules are adsorbed onto graphene flakes, it will induce a notable change on the optical contrast spectrum and thus detectable. There is a wide variety of airborne molecules (e.g., alkanes, alkenes,
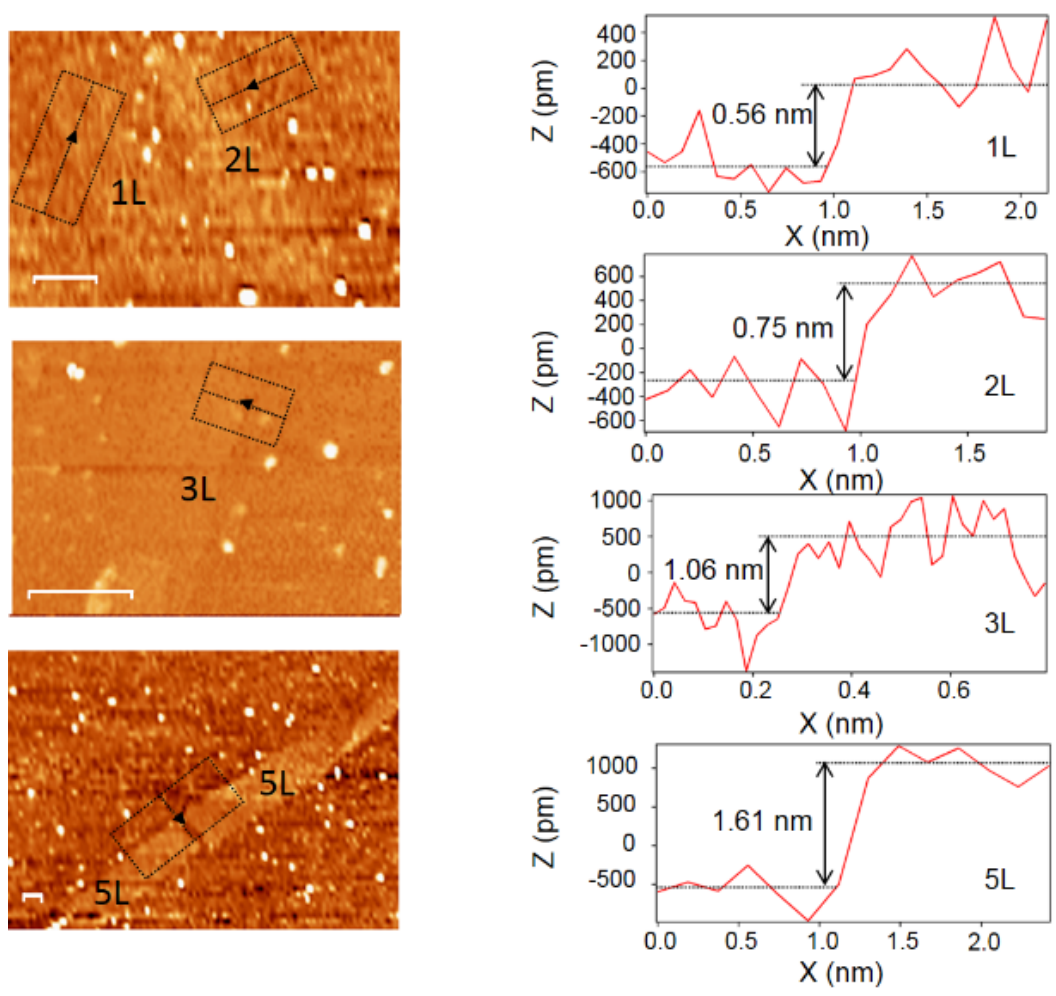

aromatics, alcohol and water) in laboratory environment that could potentially adsorb onto graphene ${ }^{41}$. It is impractical to quantify every molecule species. To provide a guiding idea about how the adsorption of airborne molecules could impact the optical contrast of graphene, we simulated the optical contrast spectra of monolayer graphene on $\mathrm{Au}$, with the adsorption of various thickness amorphous carbon film (Figure 7), which is closely relevant, as carbonaceous molecules are one of the major sources of airborne molecules.

Figure 6. (Left) AFM images and (right) step profiles of (top-bottom) 1, 2, 3, and 5 layers of the graphene flakes shown in Figure 2. The height profiles are averaged within the boxes marked in the AFM images. Arrows indicate the directions of the height profiles. All scale bars correspond to $1 \mu \mathrm{m}$.

Figure 7 a demonstrates how the contrast spectrum evolves with the thickness of amorphous carbon ( 0 to $2 \mathrm{~nm}$ from bottom to top, at $0.2 \mathrm{~nm}$ incremental step) adsorbed on the surface of a monolayer graphene deposited on $100 \mathrm{~nm} \mathrm{Au}$ substrate. It is obvious the contrast increases with the thickness of amorphous 
carbon. The wavelength of the maximum contrast slightly blueshifts initially, but slows down with further increase of thickness. The trend can be visualized more clearly in Figure $7 \mathrm{~b}$, where the maximum contrast (circles) and the peak wavelength (squares) are plotted against the thickness of amorphous carbon. The maximum contrast increases linearly with thickness $(c=3.9 t+2.17, t$ is the carbon thickness). The peak wavelength blueshifts following an exponential decay trend $\left(\lambda=485+9.78 e^{-2.2 t}\right)$. We can approximately estimate the sensitivity of the structure composed of a monolayer graphene film on 100 $\mathrm{nm} \mathrm{Au}$. The contrast detection limit of the optical system is about $0.5 \%$, estimated from the signal noise and experimental uncertainty level (Figure 3). Based on Figure 7b, this is equivalent to an average coverage of $0.13 \mathrm{~nm}$ amorphous carbon, about the thickness of one third monolayer graphene.

To test the idea of detecting airborne contamination molecules on graphene, we stored the samples in unsealed plastic boxes and kept them in ambient environment (initially the clean samples were kept in nitrogen-filled desiccators). In this way, the samples were allowed to contact air, but avoided being significantly contaminated by dust. We repeatedly measured the optical contrast spectra after 2,7 , and 21 days, respectively. The results of the measured maximum positive contrast are shown in Figure 8a, which clearly demonstrate that the contrast of the exposed samples increased considerably in comparison to the original clean samples. This is more evident when we plot the contrast increments during different period of time intervals, i.e., 0-2 days, 2-7 days and 7-21 days, respectively, as shown in Figure 8b (the increment between ' $x-y$ ' days is the contrast difference between the ' $y$ days' and the ' $x$ days' in Figure 8a).
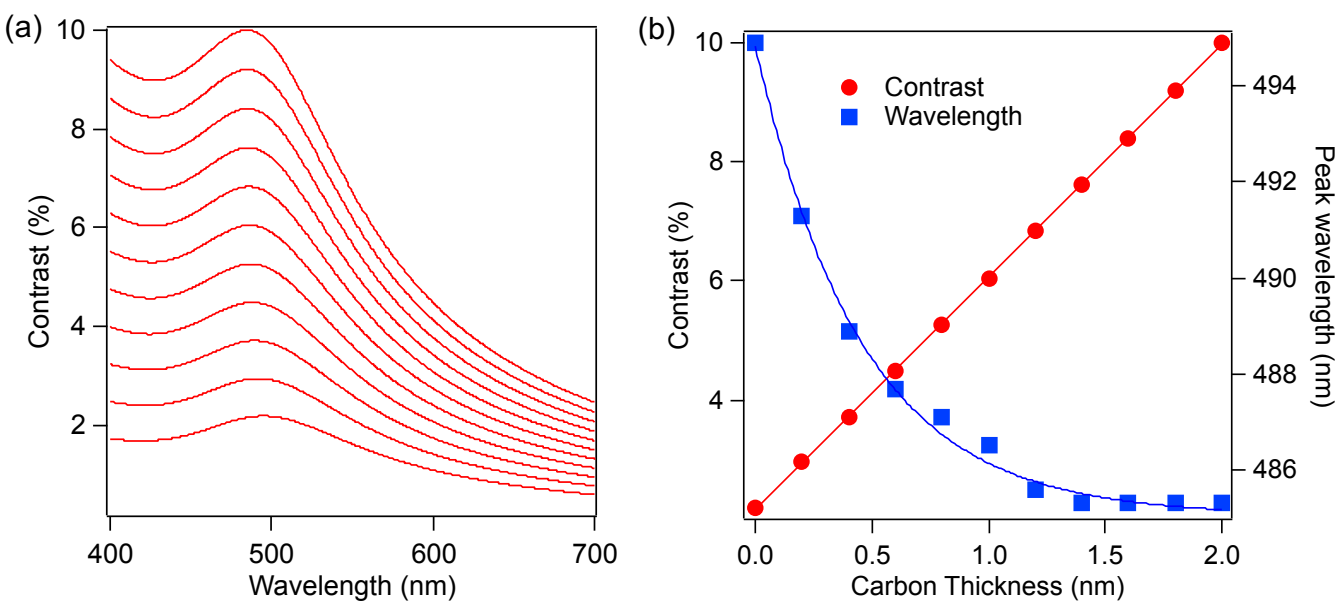
Figure 7. (a) Simulated contrast spectra of 0-2 $\mathrm{nm}$ (from bottom to top, $0.2 \mathrm{~nm}$ increment) amorphous carbon films adsorbed on monolayer graphene deposited on $100 \mathrm{~nm} \mathrm{Au}$. (b) The maximum contrast and the corresponding peak wavelength extracted from the contrast spectra in (a), as a function of the thickness of amorphous carbon. The refractive index of amorphous carbon is adopted from ref.42.

The observed contrast increase is caused by the physisorption of airborne hydrocarbon molecules. Graphene and other 2D materials are known to adsorb airborne molecules in ambient environment, as demonstrated in wettability and electrochemical studies ${ }^{4,4348}$. As shown in Figure 8b, initially the contrast increased notably after storage in ambient air for 2 days, after that the contrast increase slowed down considerably and almost plateaued after about one week, as indicated by the dashed guide line (blue). Such a time evolution trend is consistent with those reported in wettability studies ${ }^{4,4546}$, where the increase of the water contact angle exhibits a similar nonlinear behavior. The increments during the period of 721 days are small, below the detection limit $(\sim 0.5 \%)$, as the physisorption rate is drastically reduced after prolonged exposure in the air ${ }^{46}$.

A close examination of the contrast increment during the first 2 days reveals an interesting pattern: the contrast increment approximately scales with graphene thickness. Thicker films experienced larger increments, as indicated by the dashed red line. This is an intriguing observation, suggesting that the physisorption rate of airborne molecules on graphene at the early stage is not uniform: thicker films attracted more hydrocarbon molecules, therefore acquired a larger increment in contrast. If all graphene flakes had equal coverages of molecules, the contrast increment would be approximately the same for each film (for few layer graphene, the contrast increment is linear with increased layer thickness). The contrast of the ' $0 \sim 2$ days' of the $3 \mathrm{~L}$ film is unusually high. This could be caused by sample drifting during the measurement, as the flake is quite small. If it were in the normal range (dashed box) guided by the red line, the increment of the 3L film during the period of ' $2 \sim 7$ ' days would be in line with the trends of other flakes, as indicated by the dashed box. 
Atomically-thin 2D materials are excellent absorbent for many molecules, which renders them promising platforms for sensors ${ }^{474851,52}$. Molecules can attach to a solid surface by chemisorption or physisorption. Chemisorption is a strong interaction, usually involving specific chemical effects. In contrast, physisorption is a weak adhesion due to Van der Waals (vdW) interaction. Aromatic hydrocarbons can attach strongly to $2 \mathrm{D}$ materials due to strong $\pi-\pi$ interaction, which was demonstrated to be stronger on monolayers than on multilayers ${ }^{47}$. Our results are consistent with recent investigations on the effect of airborne contamination molecules on the wettability property of graphene ${ }^{4146,49}$, in which the vdW interaction is shown to be the dominant mechanism, which increases with graphene thickness up to 4-6 layers ${ }^{4+49}$.

Here we have demonstrated that the high sensitivity of the optical contrast spectroscopy allows to detect the adsorption of airborne molecules on graphene, hence can reveal whether graphene is contaminated, which is a piece of critical information central to a wide range of graphene applications, such as wettability, catalysis, adhesion, charge doping and carrier mobility. Graphene on Au substrate only has a modest contrast. The sensitivity can be significantly improved on high-contrast substrates, such as $90 \mathrm{~nm}$ $\mathrm{SiO}_{2} / \mathrm{Si}$ substrates, which has a contrast gradient of $17.7 \% / \mathrm{nm}$ for amorphous carbon on monolayer graphene (Figure S3). This will enable the detection of the adsorption of an average layer of $0.028 \mathrm{~nm}$ amorphous carbon (assuming the detection limit remains at $0.5 \%$ ). The contrast spectroscopy also can be used to detect a small variation of the refractive index (RI) of the surrounding medium. For monolayer graphene deposited on $90 \mathrm{~nm} \mathrm{SiO} / \mathrm{Si}$ substrate, simulations (Fig.S4 in SI) indicate that the contrast changes at a rate of $309 \%$ per refractive index unit (RIU), which corresponds to a sensitivity of $1.6 \times 10^{-3}$ RIU, based on $0.5 \%$ contrast detection limit. This is about one order of magnitude better than the RI sensitivity $\left(\sim 10^{-2}\right.$ RIU) of most localized surface plasmon resonance (LSPR) sensors ${ }^{s_{1}}$. Such a high sensitivity can be exploited to develop ultrasensitive molecular sensors. Graphene and a wide variety of 2D materials are newly-emerged platforms for sensors ${ }^{48.253}$, with applications in broad areas, such as humidity, gas and protein sensing. Previously many graphene sensors are based on rather complicated 
protocols, relying on the measurements of electrical conductance or the Forster energy transfer in fluorescence ${ }^{5253}$. The optical contrast method demonstrated here is a much simpler process, which is sensitive, fast, contactless, noninvasive, and can be ultimately miniaturized, as such, it provides an exciting new paradigm for sensors.
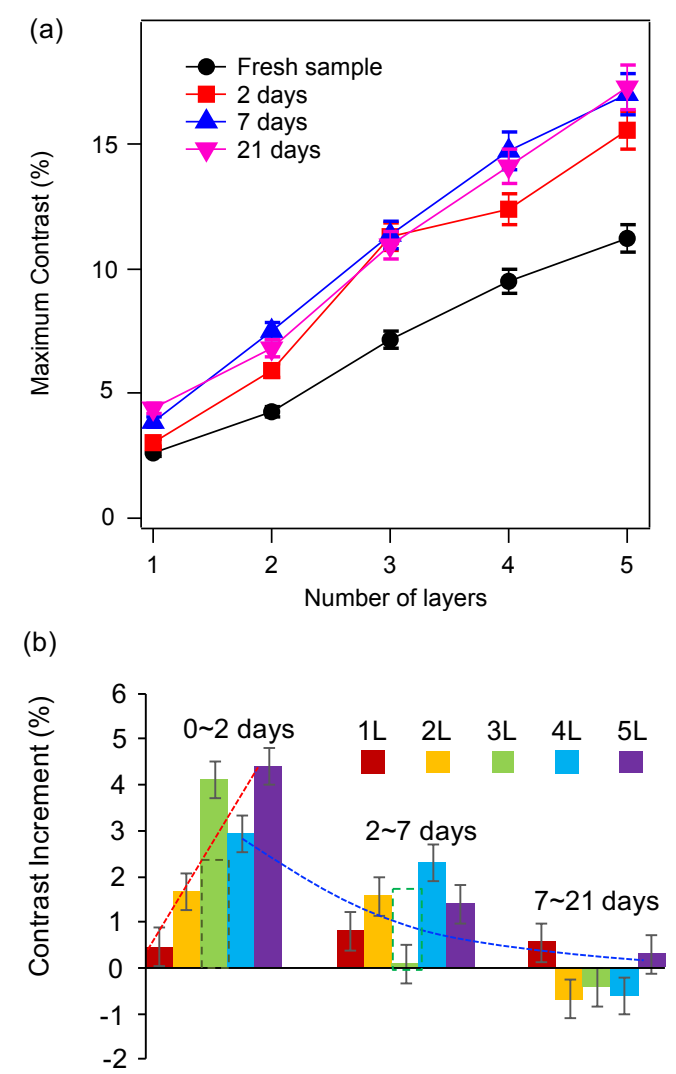

Figure 8. (a) Measured maximum optical contrast of freshly-prepared graphene flakes and after they were exposed in ambient air for 2, 7 and 21 days. (b) The increments of the optical contrast of the graphene flakes after exposure to air. Dashed lines are for guidance. Standard error bars are presented.

\section{CONCLUSIONS}

In summary, here we report a high-resolution optical contrast measurement of few-layer graphene films on bulk Au surface, using a 100× magnification objective. This provides a sub-micron spatial resolution, enabling the accurate identification of the thickness of graphene flakes as small as half a micrometre (the diffraction limit of the $100 \times$ magnification objective) in lateral size, which is significantly relevant in 
research and applications requiring high-quality pristine graphene flakes and in the development of miniaturized graphene nanodevices. The optical contrast of graphene on bulk Au surface is much lower than that reported on oxidized silicon substrate, with a maximum optical contrast of about $2.2 \%$ for a monolayer graphene film (measured by an objective with $\mathrm{NA}=0.9$ ). This is due to the high reflectivity of the gold surface, a feature shared by all reflective metals. The experimental results are found to match excellently with theoretically calculated results, when the effects of different incident angles and polarizations are appropriately taken into account. This is necessary when a high magnification objective is used. We further demonstrate that the high sensitivity of the optical contrast spectroscopy can detect the adsorption of a sub-monolayer airborne molecule and reveal whether graphene films are contaminated. In the future, by functionalizing 2D films with anchoring molecules that can only bind a specific set of molecules, such as the antigen-antibody strategies widely adopted in plasmonic sensors, one will be able to develop smart sensors that not only detect the adsorption of molecules, but reveal the identity of the adsorbed molecules as well. Though the results were demonstrated for graphene on gold, the principles can be readily generalized to any $2 \mathrm{D}$ materials and substrate. This opens new avenues for exciting novel applications of the abundant variety of 2D materials.

\section{MATERIALS AND METHODS}

The samples were prepared by consecutively depositing $10 \mathrm{~nm}$ titanium and $100 \mathrm{~nm}$ gold onto $\mathrm{Si} / \mathrm{SiOx}$ substrate by UHV magnetron sputtering. Graphene flakes were exfoliated onto the Au films immediately after they were taken out from the sputtering chamber and the optical contrast spectra were measured soon after the exfoliation. This minimizes the sample's exposure time to ambient air, mitigating the chance of contamination. The flakes were prepared using mechanical exfoliation from a natural graphite crystal (purchased from NGS Natur-graphit GmbH). It was cleaved with a high-tack, low-stain cello-tape to produce few-layer clean flakes, and pressed onto the freshly-sputtered gold surface before the tape was removed. Graphene flakes were identified with $100 \times$ Olympus objective lens (NA=0.9). Bright-field 
reflection spectra were measured using an Olympus microscope (model BX51) coupled to a QE65 Ocean Optics spectrometer, with the white light source of the microscope (12V 100W halogen lamp). An excitation laser of $532 \mathrm{~nm}$ with a power of $1 \mathrm{~mW}$ was used for Raman measurements. The signal was collected through a back-reflection configuration and coupled to a Jobin Yvon HR640 Raman spectrometer. AFM measurements were carried out in tapping mode on a Digital Instruments, Nanoscope IIIa. The data were processed with WSxM 4.0 $0^{54}$. The step heights were analyzed with Nanoscope Analysis 1.5 .

The optical contrast is calculated using the following equation:

$$
C=1-\frac{R_{G r}}{R_{A u}}
$$

where $C$ is the optical contrast, $\mathrm{R}_{\mathrm{Au}}$ is the reflectance of light on bare gold substrates, and $\mathrm{R}_{\mathrm{Gr}}$ is the reflectance of light on the Au substrate covered with a graphene flake. The contrast is positive (negative) when the addition of a graphene flake reduces (increases) the reflectance of light on Au substrate.

\section{SUPPORTING INFORMATION}

General formulae for the calculation of the reflectivity of light incident through an objective, comparison between the measured and the calculated reflectivity spectra of light from the surface of a monolayer graphene deposited on $100 \mathrm{~nm} \mathrm{Au} \mathrm{substrate,} \mathrm{calculated} \mathrm{contrast} \mathrm{spectra}$ of a monolayer graphene deposited on a Si substrate coated with $90 \mathrm{~nm} \mathrm{SiO}_{2}$, as a function of the thickness of adsorbed amorphous carbon on top, calculated contrast spectra of a monolayer graphene deposited on a Si substrate coated with $90 \mathrm{~nm} \mathrm{SiO}$, as a function of the refractive index of the surrounding medium.

\section{AUTHOR INFORMATION}

\section{Corresponding Author}


*E-mail: f.huang@qub.ac.uk

\section{Author Contributions}

The manuscript was written through contributions of all authors. All authors have given approval to the final version of the manuscript.

\section{Funding Sources}

This work was supported by the Department of Employment and Learning of Northern Ireland (DEL) and UK EPSRC (ref. EP/N025938/1)

\section{REFERENCES}

1. Novoselov, K. S.; Geim, A. K.; Morozov, S. V.; Jiang, D.; Zhang, Y.; Dubonos, S. V.; Grigorieva, I. V.; Firsov, A. A. Electric Field Effect in Atomically Thin Carbon Films. Science. 2004, 306, 666-669.

2. Cooper, D. R.; D’Anjou, B.; Ghattamaneni, N.; Harack, B.; Hilke, M.; Horth, A.; Majlis, N.; Massicotte, M.; Vandsburger, L.; Whiteway, E.; Yu, V. Experimental Review of Graphene. ISRN Condens. Matter Phys. 2012, 2012, 1-56.

3. Geim, A. K.; Novoselov, K. S. The Rise of Graphene. Nat. Mater. 2007, 6, 183-191.

4. Chen, J. H.; Jang, C.; Xiao, S.; Ishigami, M.; Fuhrer, M. S. Intrinsic and Extrinsic Performance Limits of Graphene Devices on $\mathrm{SiO}_{2}$. Nat. Nanotechnol. 2008, 3, 206-209.

5. Jung, G. S.; Yeo, J.; Tian, Z.; Qin, Z; Buehler, M. J. Unusually Low and Density-Insensitive Thermal Conductivity of Three-Dimensional Gyroid Graphene. Nanoscale. 2017, 9, 13477-13484.

6. Bonaccoroso, F.; Sun, Z.; Hasan, T.; Ferrari, A. C. Graphene Photonics and Optoelectronics. Nat. Photonics. 2010, 4, 611-622.

7. Bao, Q.; Loh, K. P. Graphene Photonics, Plasmonics, and Broadband Optoelectronic Devices. ACS Nano. 2012, 6, 3677-3694.

8. Jung, D. Y.; Yang, S. Y.; Park, H.; Shin, W. C.; Oh, J. G.; Cho, B. J.; Choi, S. Y. Interface Engineering for High Performance Graphene Electronic Devices. Nano Convergence. 2015, 2, 1-17. 
9. Siokou, A.; Ravani, F.; Karakalos, S.; Frank, O.; Kalbac, M.; Galiotis, C. Surface Refinement and Electronic Properties of Graphene Layers Grown on Copper Substrate: an XPS, UPS and EELS Study. Appl. Surf. Sci. 2011, 257, 9785-9790.

10. Dahal, A.; Batzill, M. Graphene-Nickel Interfaces: A Review. Nanoscale. 2014, 6, 2548-2562.

11. Choi, S. H.; Kim, Y. L.; Byun, K. M. Graphene-on-Silver Substrates for Sensitive Surface Plasmon Resonance Imaging Biosensors. Opt. Express. 2011, 19, 458-466.

12. Wu, L.; Chu, H. S.; Koh, W. S.; Li, E. P. Highly Sensitive Graphene Biosensors Based on Surface Plasmon Resonance. Opt. Express. 2010, 14, 14395-14400.

13. Cai, J.; Ruffieux, P.; Jaafar, R.; Bieri, M.; Braun, T.; Blankenburg, S.; Muoth, M.; Seitsonen, A. P.; Saleh, M.; Feng, X.; Mullen, K. Atomically Precise Bottom-Up Fabrication of Graphene Nanoribbons. Nature. 2010, 466, 470-473.

14. Zhou, X.; Qi, Y.; Shi, J.; Niu, J.; Liu, M.; Zhang, G.; Li, Q.; Zhang, Z.; Hong, M.; Ji, Q.; Zhang, Y. Modulating the Electronic Properties of Monolayer Graphene Using a Periodic Quasi-One-Dimensional Potential Generated by Hex-Reconstructured Au (111). ACS Nano. 2016, 10, 7550-7557.

15. Xu, Y.; He, K. T.; Schmucker, S. W.; Guo, Z.; Koepke, J. C.; Wood, J. D.; Lyding, J. W.; Aluru, N. R. Inducing Electronic Changes in Graphene Through Silicon (100) Substrate Modification. Nano Lett. 2011, 11, 2735-2742.

16. Gierz, I.; Riedl, C.; Starke, U.; Ast, C. R.; Kern, K. Atomic Hole Doping of Graphene. Nano Lett. 2008, 8, 4603-4607.

17. Panchakarla, L. S.; Subrahmanyam, K. S.; Saha, S. K.; Govindaraj, A.; Krishnamurthy, H. R.; Waghmare, U. V.; Rao, C. N. R. Synthesis, Structure, and Properties of Boron- and Nitrogen-Doped Graphene. Adv. Mater. 2009, 21, 4726-4730.

18. Novoselov, K. S.; Fal, V. I.; Colombo, L.; Gellert, P. R.; Schwab, M. G.; Kim, K. A Roadmap for Graphene. Nature. 2012, 490, 192-200. 
19. Choi, K.; Lee, Y. T.; Im, S. Two-Dimensional van der Waals Nanosheet Devices for Future Electronics and Photonics. Nano Today. 2016, 11, 626-643.

20. Han, W.; Kawakami, R. K.; Gmitra, M.; Fabian, J. Graphene Spintronics. Nat. Nanotechnol. 2014, 9 , 794-807.

21. Shearer, C. J.; Slattery, A. D.; Stapleton, A. J.; Shapter, J. G.; Gibson, C. T. Accurate Thickness Measurement of Graphene. Nanotechnol. 2016, 27, 125704.

22. Ferrari, A. C.; Meyer, J. C.; Scardaci, V.; Casiraghi, C.; Lazzeri, M.; Mauri F.; Piscanec, S.; Jiang, D.; Novoselov, K. S.; Roth, S.; Geim, A. L. Raman Spectrum of Graphene and Graphene Layers. Phys. Rev. Lett. 2006, 97, 187401-187404.

23. Calizo, I.; Balandin, A. A.; Bao, W.; Miao, F.; Lau, C. N. Temperature Dependence of the Raman Spectra of Graphene and Graphene Multilayers. Nano Lett. 2007, 7, 2645-2649.

24. Ni, Z. H.; Yu, T.; Lu, Y. H.; Wang, Y. Y.; Feng, Y. P.; Shen, Z. X. Uniaxial Strain on Graphene: Raman Spectroscopy Study and Band-Gap Opening. ACS Nano. 2008, 2, 2301-2305.

25. Lenski, D. R.; Fuhrer, M. S. Raman and Optical Characterization of Multilayer Turbostratic Graphene Grown via Chemical Vapor Deposition. J. Appl. Phys. 2011, 110, 013720-013724.

26. Gorbachev, R. V.; Riaz, I.; Nair, R. R.; Jalil, R.; Britnell, L.; Belle, B. D.; Hill, E. W.; Novoselov, K. S.; Watanabe, K.; Taniguchi, T.; Geim, A. K. Hunting for Monolayer Boron Nitride: Optical and Raman Signatures. Small. 2011, 7, 465-468.

27. Splendiani, A.; Sun, L.; Zhang, Y.; Li, T.; Kim, J.; Chim, C. Y.; Galli, G.; Wang, F. Emerging Photoluminescence in Monolayer $\mathrm{MoS}_{2}$. Nano Lett. 2010, 10, 1271-1275.

28. Cai, Q.; Scullion, D.; Falin, A.; Watanabe, K.; Taniguchi, T.; Chen, Y.; Santos, E. J.; Li, L. H. Raman Signature and Phonon Dispersion of Atomically Thin Boron Nitride. Nanoscale. 2017, 9, 3059-3067. 29. Ni, Z. H.; Wang, H. M.; Kasim, J.; Fan, H. M.; Yu, T.; Wu, Y. H.; Feng, Y. P.; Shen, Z. X. Graphene Thickness Determination Using Reflection and Contrast Spectroscopy. Nano Lett. 2007, 7, 2758-2763. 
30. Wlasny, I.; Dabrowski, P.; Klusek, Z. Optical Contrast of Single- and Multi-Layer Graphene Deposited on a Gold Substrate, arXiv:1102.4953.

31. Born, M; Wolf, E. Principles of Optics, 7th ed., University Press: Cambridge, 1999.

32. Blake, P.; Hill, E. W.; Castro Neto, A. H.; Novoselov, K. S.; Jiang, D.; Yang, R.; Booth, T. J., Geim, A. K. Making Graphene Visible. Appl. Phys. Lett. 2007, 91, 63124-63127.

33. Rakić, A. D.; Djurišic, A. B.; Elazar, J. M.; Majewski, M. L. Optical Properties of Metallic Films for Vertical-Cavity Optoelectronic Devices. Appl. Opt. 1998, 37, 5271-5283.

34. Wang, X.; Chen, Y. P.; Nolte, D. D. Strong Anomalous Optical Dispersion of Graphene: Complex Refractive Index Measured by Picometrology. Opt. Express. 2008, 16, 22105-22112.

35. Falkovsky, L. A. Optical Properties of Graphene. J. Phys.: Conf. Ser. 2008, 129, 1-7.

36. Ni, Z.; Liu, L.; Wang, Y.; Zheng, Z.; Li, L.J.; Yu, T.; Shen, Z. G-Band Raman Double Resonance in Twisted Bilayer Graphene: Evidence of Band Splitting and Folding. Phys. Rev. B. 2009, 80, 125404125408.

37. Casiraghi, C. Doping Dependence of the Raman Peaks Intensity of Graphene Close to the Dirac Point. Phys. Rev. B. 2009, 80, 233407.

38. Lee, J.; Shim, S.; Kim, B.; Shin, H. S. Surface-Enhanced Raman Scattering of Single- and Few-Layer Graphene by the Deposition of Gold Nanoparticles. Chem. Eur. J. 2011, 17, 2381-2387.

39. Hawaldar, R.; Merino, P.; Correia, M. R.; Bdikin, I.; Grácio, J.; Méndez, J.; Martín-Gago, J. A.; Singh, M.K. Large-Area High-Throughput Synthesis of Monolayer Graphene Sheet by Hot Filament Thermal Chemical Vapor Deposition. Sci. Rep. 2012, 2, 682-690.

40. Lui, C. H.; Liu, L.; Mak, K. F.; Flynn, G. W.; Heinz, T. F. Ultraflat Graphene. Nature. 2009, 462, $339-341$.

41. Li, Z.; Wang, Y.; Kozbial, A.; Shenoy, G.; Zhou, F.; McGinley, R.; Ireland, P.; Morganstein, B.; Kunkel, A.; Surwade, S. P.; Li, L. Effect of Airborne Contaminants on the Wettability of Supported Graphene and Graphite. Nat. Mater. 2013, 12, 925-931. 
42. Duley, W. W., Refractive Indices for Amorphous Carbon. Astrophys. J. 1984, 287, 694-696.

43. Velický, M.; Bradley, D. F.; Cooper, A. J.; Hill, E. W.; Kinloch, I. A.; Mishchenko, A.; Novoselov, K. S.; Patten, H. V.; Toth, P. S.; Valota, A. T.; Worrall, S. D. Electron Transfer Kinetics on Mono- and Multilayer Graphene. ACS Nano. 2014, 8, 10089-10100.

44. Velický, M.; Bissett, M. A.; Toth, P. S.; Patten, H. V.; Worrall, S. D.; Rodgers, A. N.; Hill, E. W.; Kinloch, I. A.; Novoselov, K. S.; Georgiou, T.; Britnell, L. Electron Transfer Kinetics on Natural Crystals of $\mathrm{MoS}_{2}$ and Graphite. Phys. Chem. Chem. Phys. 2015, 17, 17844-17853.

45. Lai, C. Y.; Tang, T. C.; Amadei, C. A.; Marsden, A. J.; Verdaguer, A.; Wilson, N.; Chiesa, M. A Nanoscopic Approach to Studying Evolution in Graphene Wettability. Carbon. 2014, 80, 784-792.

46. Aria, A. I.; Kidambi, P. R.; Weatherup, R. S.; Xiao, L.; Williams, J. A.; Hofmann, S. Time Evolution of the Wettability of Supported Graphene Under Ambient Air Exposure. J. Phys. Chem. C. 2016, 120, 2215-2224.

47. Cai, Q.; Du, A.; Gao, G.; Matei, S.; Cowie, B. C.; Qian, D.; Zhang, S.; Lu, Y.; Fu, L.; Taniguchi, T.; Huang, S. Molecule-Induced Conformational Change in Boron Nitride Nanosheets with Enhanced Surface Adsorption. Adv. Funct. Mater. 2016, 26, 8202-8210.

48. Cai, Q.; Matei, S.; Watanabe, K.; Taniguchi, T.; Huang, S.; Chen, Y.; Li, L. H. Boron Nitride Nanosheet-Veiled Gold Nanoparticles for Surface-Enhanced Raman Scattering. ACS Appl. Mater. Interfaces. 2016, 8, 15630-15636.

49. Shih, C. J.; Wang, Q. H.; Lin, S.; Park, K. C.; Jin, Z.; Strano, M. S.; Blankschtein, D. Breakdown in the Wetting Transparency of Graphene. Phys. Rev. Lett. 2012, 109, 176101-176105.

50. Rafiee, J.; Mi, X.; Gullapalli, H.; Thomas, A. V.; Yavari, F. Shi, Y.; Ajayan, P. M.; Koratkar, N. A. Wetting Transparency of Graphene. Nat. Mater. 2012, 11, 217-222.

51. Anker, J. N.; Hall, W. P.; Lyandres, O.; Shah, N. C.; Zhao, J.; Van Duyne, R. P. Biosensing with Plasmonic Nanosensors. Nat. Mater. 2008, 7, 442-453. 
52. Malic, E.; Appel, H.; Hoffman, O. T.; Rubio, A. Forster-Induced Energy Transfer in Functionalized Graphene. J. Phys. Chem. C. 2014, 118, 9283-9289.

53. Neri, G., Thin 2D: The New Dimensionality in Gas Sensing. Chemosensors. 2017, 5, 21.

54. Horcas, I.; Fernández, R.; Gomez-Rodriguez, J. M.; Colchereo, J.; Gomez-Herrero, J. W. S. X. M.;

Baro, A. M. WSXM: A Software for Scanning Probe Microscopy and a Tool for Nanotechnology. Rev. Sci. Instrum. 2007, 78, 013705-8. 